\title{
Semiconducting Graphene from Highly Ordered Substrate Interactions
}

\author{
M. S. Nevius, ${ }^{1}$ M. Conrad, ${ }^{1}$ F. Wang, ${ }^{1}$ A. Celis, ${ }^{2,3}$ M. N. Nair, ${ }^{4}$ A. Taleb-Ibrahimi, ${ }^{4}$ A. Tejeda, ${ }^{2,3}$ and E. H. Conrad ${ }^{1, *}$ \\ ${ }^{1}$ The Georgia Institute of Technology, Atlanta, Georgia 30332-0430, USA \\ ${ }^{2}$ Laboratoire de Physique des Solides, Université Paris-Sud, CNRS, UMR 8502, F-91405 Orsay Cedex, France \\ ${ }^{3}$ Synchrotron SOLEIL, L'Orme des Merisiers, Saint-Aubin, 91192 Gif sur Yvette, France \\ ${ }^{4}$ URI CNRS/Synchrotron SOLEIL, Saint-Aubin, 91192 Gif sur Yvette, France
}

(Received 3 May 2015; published 21 September 2015)

\begin{abstract}
While numerous methods have been proposed to produce semiconducting graphene, a significant band gap has never been demonstrated. The reason is that, regardless of the theoretical gap formation mechanism, subnanometer disorder prevents the required symmetry breaking necessary to make graphene semiconducting. In this work, we show for the first time that semiconducting graphene can be made by epitaxial growth. Using improved growth methods, we show by direct band measurements that a band gap greater than $0.5 \mathrm{eV}$ can be produced in the first graphene layer grown on the $\mathrm{SiC}(0001)$ surface. This work demonstrates that order, a property that remains lacking in other graphene systems, is key to producing electronically viable semiconducting graphene.
\end{abstract}

PACS numbers: 73.22.Pr, 79.60.Bm, 79.60.Jv, 81.05.ue

It is well known that the first graphene layer grown on the $\mathrm{SiC}(0001)$ surface is not electronic graphene. That is, the first "buffer" graphene layer does not show the linear dispersing $\pi$ bands (Dirac cone) expected at the $K$ point of metallic graphene [1-3]. The lack of $\pi$ bands in experimental band maps of the buffer layer [2] supported the theoretical conclusion that sufficiently strong covalent bonds between the buffer layer and the $\mathrm{SiC}$ interface would push the graphene $\pi$ bands below the $\mathrm{SiC}$ valence band maximum [4,5]. Aside from these very early studies, research on the $\mathrm{SiC}$ graphene buffer layer faded and was subsequently eclipsed by a wide variety of other unsuccessful ideas to open a band gap in exfoliated or chemical vapor deposition (CVD)-grown graphene [6].

One method to open a band gap in graphene is by periodic bonding to either all $A$ or all $B$ sites, which breaks graphene's chiral symmetry (referred to as graphene functionalization). The buffer graphene, commensurately bonded to the $\mathrm{SiC}(0001)$ surface, should have been an excellent example of a functionalized system. Despite the buffer graphene's potential to be functionalized by a commensurate and, most importantly, ordered array of $\mathrm{Si}$ or $\mathrm{C}$ atoms at the $\mathrm{SiC}$ surface, there was instead a major research shift to functionalize CVD-grown graphene. As of this writing, no functionalized graphene, or graphene modified by any other proposed method, has been developed that produces a workable semiconducting form of graphene. The problem with these methods is the inherent disorder introduced by the functionalization $[7,8]$ and growth processes [6]. In fact, the lack of a graphene band gap was the motivation to shift research to metal dichalcogenides despite the inability to grow them at the level of purity and order required for scalable electronics.

In this work, we use furnace-grown graphene to produce a structurally well-ordered buffer graphene (BG) on the
$\mathrm{SiC}(0001)$ surface. Angle-resolved photoemission (ARPES) measurements show new dispersing $\pi$ bands that are not observed in samples grown by previous methods. These bands live above the $\mathrm{SiC}$ valence band maximum near the Fermi energy $E_{F}$. The new band structure is a result of improved order caused by a higher growth temperature which, for the first time, gives rise to a well-ordered $6 \times 6$ reconstruction in surface $\mathrm{x}$-ray scattering experiments [9]. The band gap, which is $>0.5 \mathrm{eV}$, appears to be the result of the chiral symmetry breaking caused by the $6 \times 6$ reconstruction. We demonstrate that the buffer graphene layer on $\mathrm{SiC}$ is a true semiconductor, the goal of the first graphene electronics research $[10,11]$.

ARPES measurements were made at the Cassiopée beam line at the SOLEIL synchrotron. Sample temperature was held at $90 \mathrm{~K}$ for all experiments. The high-resolution Cassiopée beam line is equipped with a modified Peterson PGM monochromator with a resolution $E / \Delta E$ of 25000 for energies below $100 \mathrm{eV}$. The detector is a Scienta R4000 detector with a base resolution of $\Delta E<1 \mathrm{meV}$. The total energy and momentum resolution were $12 \mathrm{meV}$ and $0.01 \AA^{-1}$, respectively. $E_{F}$ was determined to within $20 \mathrm{meV}$ using the intensity cutoff of the molybdenum sample holder and checked against the cutoff energy of the $k$-integrated $E(k)$ for each sample. The $\mathrm{SiC}$ substrates were $n$-doped CMP polished on-axis $4 \mathrm{H}-\mathrm{SiC}(0001)$. Graphene was grown in a controlled silicon sublimation furnace [12]. Graphene growth is a function of temperature, time, and crucible geometry that sets the silicon vapor pressure. With the current crucible design, a monolayer (MG) graphene film will grow in $20 \mathrm{~min}$ at $1520^{\circ} \mathrm{C}$. Using the same crucible, the semiconducting BG discussed in this Letter will grow in the same time at a temperature $160^{\circ} \mathrm{C}$ lower than MG. Growing $20^{\circ} \mathrm{C}$ lower 
than the optimum buffer temperature gives the same $(6 \sqrt{3} \times 6 \sqrt{3}) R 30^{\circ}$ LEED pattern (subsequently referred to as $6 \sqrt{3}$ ) as the optimum buffer film but will not show the gapped $\pi$ bands discussed below.

Early ARPES work on the UHV-grown $6 \sqrt{3}$ reconstructed $\mathrm{SiC}(0001)$ surface (referred to as the graphene buffer layer in later literature) found that two nondispersing states $g_{1}$ and $g_{2}$ at -0.5 and $-1.6 \mathrm{eV}$ were the only band features between $E_{F}$ and the $\mathrm{SiC}$ valence band maximum [2]. These states were interpreted as localized MottHubbard states hybridized from $\mathrm{SiC}$ surface dangling bonds. We can reproduce these states by heating the $\mathrm{SiC}$ $20^{\circ} \mathrm{C}$ cooler than the optimal buffer growth temperature. Figure 1(a) shows an ARPES cut through the graphene $K$ point from this "subbuffer" film. The previous surface states are clearly visible. However, by heating $20^{\circ} \mathrm{C}$ higher, a new dispersing band $\bar{\epsilon}_{1}(k)$ appears [see Fig. 1(b)]. The new surface state is robust, being reproducible in multiple samples. Note that a faint linear Dirac cone appears at $k_{y}=0$. This is due to a small amount of $\mathrm{MG}(<2 \%)$ that typically nucleates at intrinsic step edges [13]. The Dirac point of the partial monolayer has the typical $n$ doping $\left(0.55 \mathrm{eV}\right.$ below $\left.E_{F}\right)$.

Another indication of the improved sample order is the quality of the MG grown above the optimum buffer. Figure 2 shows a constant energy cut through part of the Brillouin zone (BZ) of a MG film. In addition to the Dirac cone, replicas of the Dirac cone from umklapp scattering processes are also visible. All replica cones from the $K$ th $K$ point can be indexed using reciprocal lattice vectors of the SiC $6 \times 6$ unit cell: $\mathbf{G}_{K}(m, n)=m \mathbf{s}_{1}+n \mathbf{s}_{2}$, where $\left|\mathbf{s}_{1}\right|=$ $\left|\mathbf{s}_{2}\right|=\frac{1}{6}\left|\mathbf{a}_{\text {SiC }}^{*}\right|$ [see Fig. 2]. In the ordered MG films, replica

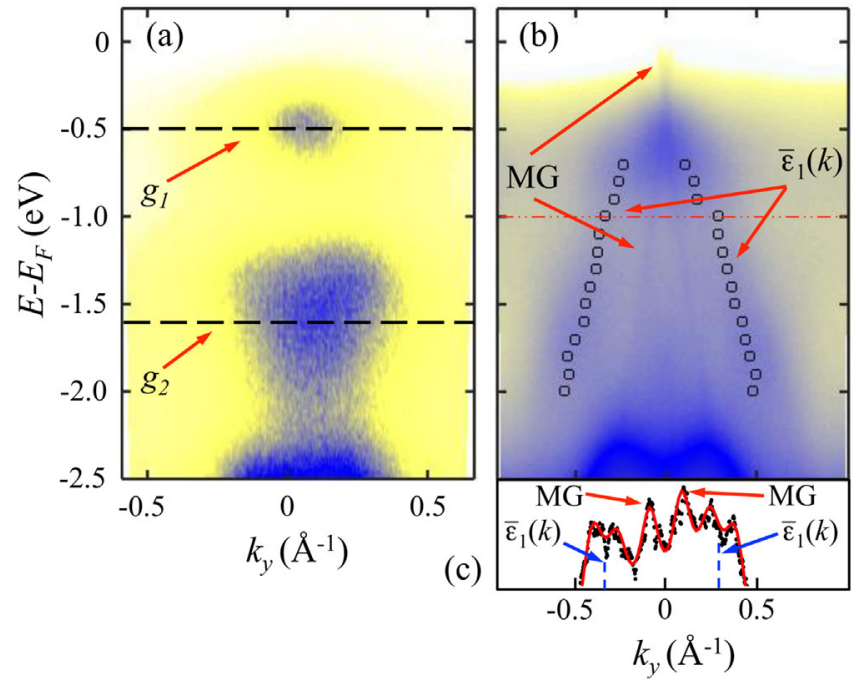

FIG. 1 (color online). (a) An ARPES cut through the graphene $K$ point of an undergrown $6 \sqrt{3}$ surface. $k_{y}$ is perpendicular to $\Gamma-K(h \nu=36 \mathrm{eV})$. The states $g_{1}$ and $g_{2}$ observed by Emtsev et al. [2] are marked. (b) The same cut as (a) for growth $20^{\circ} \mathrm{C}$ higher $(h \nu=70 \mathrm{eV})$. Circles mark the peak positions along part of the $\bar{\epsilon}_{1}$ band. (c) The MDC through the bands at $1 \mathrm{eV}$. cones are clearly seen from both first order in the $6 \times 6$ unit cell $\left(\mathbf{s}_{1}, \mathbf{s}_{2}\right)$ and from multiple scattering processes involving first order $\left(\mathbf{s}_{1}, \mathbf{s}_{2}\right)$ plus a $\mathrm{SiC} \mathbf{G}$ vector [e.g., the $\mathbf{G}_{K}(\overline{\overline{7}}, 0)$ and $\left.\mathbf{G}_{K}(\overline{\overline{7}}, 1)\right]$. Early UHV-grown samples only showed first-order replicas (i.e., $n, m=1$ ) [14]. The fact that so many ARPES replica bands are observed in these films, along with the sixth-order x-ray diffraction rods [9], testifies to the film's improved order.

Detailed ARPES measurements from these improved samples show a gapped set of $\pi$-band bands. A momentum distribution curve (MDC) through the $\pi$ band [Fig. 1(c)] shows three sets of bands: one set from the partial MG and a split pair of bands $\bar{\epsilon}_{1}(k)$. Even though the split bands have a $\Delta k$ width that is only $12 \%$ wider than the MG bands, their splitting $\left(<0.13 \AA^{-1}\right)$ and the background level make them difficult to resolve. In this work, we will refer to these bands by their average dispersion $\bar{\epsilon}_{1}(k)$. More detailed studies of these bands will be presented in a future work. Figure 3(a) shows a constant energy cut though part of the BZ of a buffer layer graphene near the $\bar{\epsilon}_{1}(k)$ band maximum. Three lobes are visible that represent a second dispersing band, $\epsilon_{2}(k)$, that is marked in the $\Gamma K M^{\prime}$ cut in Figs. 3(b) and 3(c). Again, a Dirac cone from a small amount of MG is visible. The two bands are independent of the perpendicular momentum $k_{\perp}(E)$ and therefore cannot be due to bulk bands. The tops of both bands lie $\Delta E \sim 0.5 \mathrm{eV}$ below $E_{F}$, or $1.8 \mathrm{eV}$ above the valence band maximum of $\mathrm{SiC}$ interface, indicating that the MG is semiconducting graphene with a band gap suitable for real electronics application. A schematic of the two bands is shown in Fig. 4. The $\bar{\epsilon}_{1}(k)$ band appears as a gapped $\pi$ band that disperses slower perpendicular to $\Gamma K$ than along either $\Gamma K$ or $K M$ [see Table I]. The linear part of $\bar{\epsilon}_{1}(k)$ has a velocity $v$ that is significantly lower than the Fermi velocity $v_{F}$, reducing to nearly half $v_{F}$ perpendicular to $\Gamma K$ [see Table I].

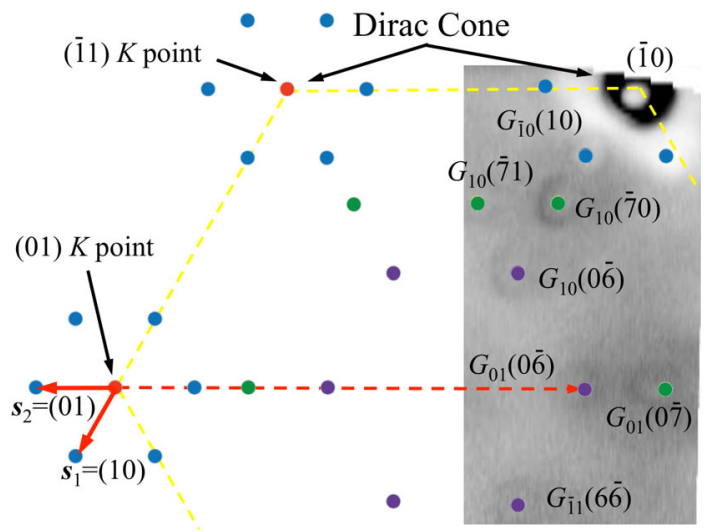

FIG. 2 (color online). The right panel is an ARPES constant energy cut through part of a MG BZ showing replica cones $\left(E-E_{F}=-1 \mathrm{eV}, h \nu=36 \mathrm{eV}\right)$. Blue dots mark single $6 \times 6$ umklapp replicas of the Dirac cones $\left(\mathbf{s}_{1}, \mathbf{s}_{2}\right)$. Umklapp scattered Dirac cones from $\mathrm{SiC} 1 \times 1$ (purple dots) and $\mathrm{SiC} 1 \times 1$ plus $6 \times 6$ reciprocal lattice vectors (green dots) are also shown. 

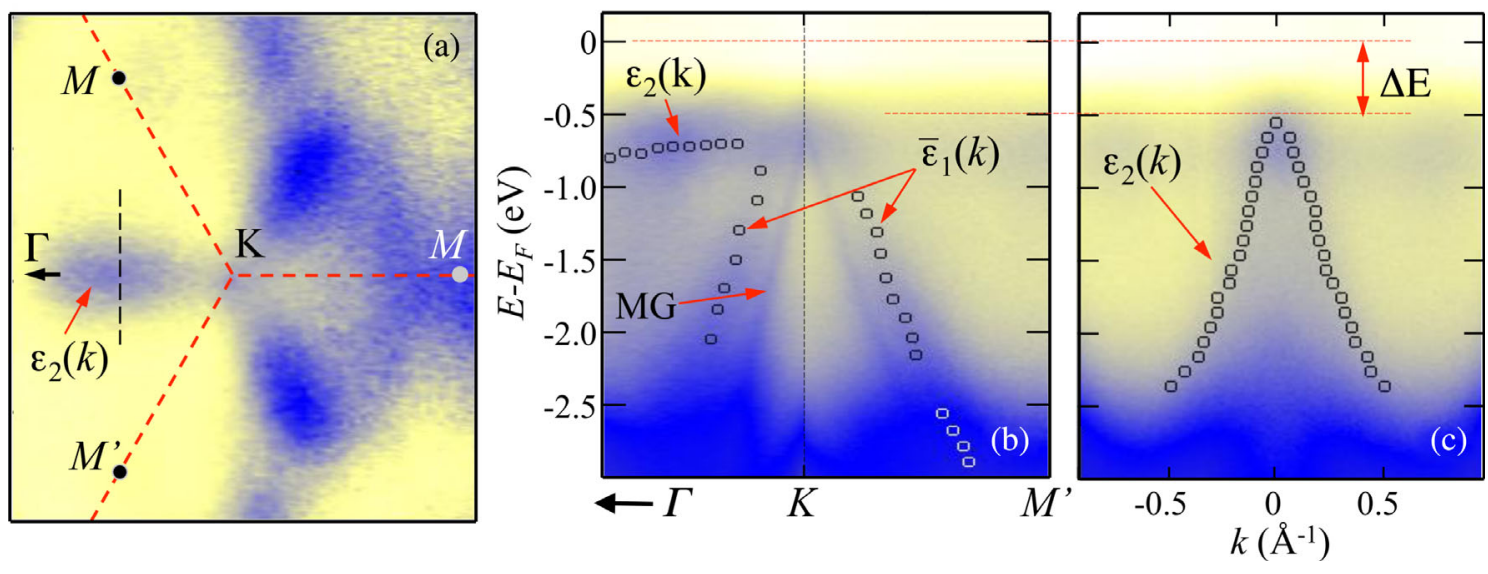

FIG. 3 (color online). (a) A constant energy cut through the graphene BZ near the $K$ point $\left(E-E_{F}=-0.41 \mathrm{eV}, h \nu=70 \mathrm{eV}\right)$. Dashed lines mark the BZ boundary. (b) A cut through the surface bands in the $\Gamma K M^{\prime}$ direction. Circles mark the peak positions along part of the $\bar{\epsilon}_{1}$ and $\epsilon_{2}$ band along with a few higher binding energy bands. A Dirac cone from a partial MG is shown. (c) A cut perpendicular to $\Gamma K$ through the $\epsilon_{2}$ band [vertical black dashed line in (a)]. Circles mark the peak positions of the $\epsilon_{2}$ band.

The $\epsilon_{2}(k)$ band is threefold symmetric, extending towards $\Gamma$ and dispersing perpendicular to $\Gamma K$. Figure 3(c) shows a cut perpendicular through the lobe in Fig. 3(a). The band velocity of $\epsilon_{2}(k)$ perpendicular to $\Gamma K$ is nearly the same as monolayer graphene [see Table I]. The $\bar{\epsilon}_{1}$ band has an effective mass $\left(m^{*}\right)$ that ranges between 0.55 and $1.5 m_{e}$, while $\epsilon_{2}$ is a light band perpendicular to $\Gamma K$ but has a smaller dispersion (larger $m^{*}$ ) along $\Gamma K$.

In a broad sense, the gapped band structure strongly suggests chiral symmetry breaking that mixes the $\pi$ bands from the $K$ and $K^{\prime}$ points [15]. Any periodic potentials that break the $A B$ symmetry in the graphene through bond formation, chemical or strain fields, or finite size effects can open a gap. Weak interactions like those in bilayer graphene produce only small gaps [16]. The strain necessary to open the observed band gaps by Kekule distortions [17] would also be large enough to tear the graphene [18], inconsistent with the $0.7 \%$ strain measured by $\mathrm{x}$-ray scattering $[9,19]$. Periodic bonding of the BG to the underlying substrate remains the most likely source of the chiral symmetry breaking.

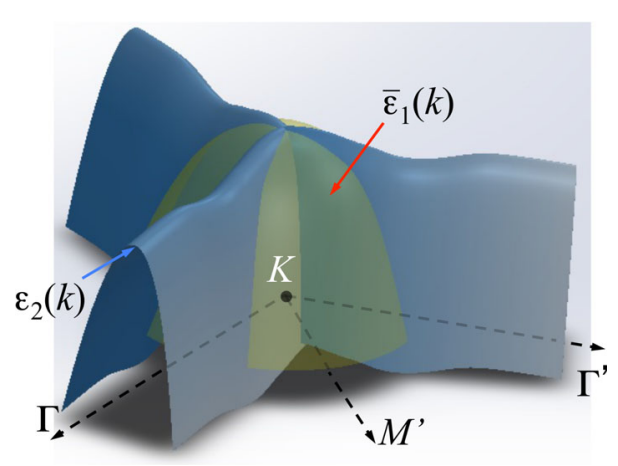

FIG. 4 (color online). A schematic of the $\bar{\epsilon}_{1}(k)$ and $\epsilon_{2}(k)$ buffer layer bands near the top of the $\pi$ bands around the $K$ point.
A theoretical understanding of the BG layer, and therefore an understanding of the origin of the observed gap, is difficult because of the excessive calculation time associated with exploring different models for the large $6 \sqrt{3}$ unit cell. Rather than calculating the full cell, early calculations instead used a strained $\sqrt{3} \times \sqrt{3} R 30 \mathrm{SiC}$ reconstruction to make calculations more tractable $[4,5]$. These calculations predicted that strong $s p^{3}$ bonds between $2 / 3$ of the interfacial $\mathrm{Si}$ atoms and the buffer graphene caused the $\pi$ bands to shift above and below the conduction band minimum and valence band maximum, respectively. The calculations also predicted a metallic, slightly delocalized, surface state near $E_{F}$ due to unbounded $\mathrm{Si}$ atoms in the interface, similar to the states observed experimentally in the earlier, less ordered samples like in Fig. 1(a) [2]. These approximate models are clearly insufficient to explain the observed bands. Only one ab initio calculation by Kim et al. [20] has calculated the band structure for the buffer using a full $6 \sqrt{3}$ cell. While the calculation was restricted to a bulk terminated interface [20], it does give some insight into the origin of the observed gap when compared to the ARPES results.

Kim et al. [20] find that about $25 \%$ of the carbon atoms in the buffer graphene are covalently bonded to $\mathrm{Si}$ atoms on

TABLE I. Band velocity and effective mass $\left(m^{*}\right)$ near the $\pi$-band maximum. $m^{*}$ is estimated assuming parabolic bands.

\begin{tabular}{lcc}
\hline \hline Band & $v / v_{F}$ & $m^{*} / m_{e}$ \\
\hline MG Dirac cone & 1.0 & $\ldots$ \\
$\bar{\epsilon}_{1}(\perp \Gamma K)$ & $0.55 \pm 0.01$ & $1.0 \pm 0.02$ \\
$\bar{\epsilon}_{1}(\Gamma K)$ & $0.63 \pm 0.1$ & $1.5 \pm 0.5$ \\
$\bar{\epsilon}_{1}(K M)$ & $0.80 \pm 0.1$ & $0.55 \pm 0.05$ \\
$\epsilon_{2}(\perp \Gamma K)$ & $0.98 \pm 0.07$ & $0.25 \pm 0.02$ \\
$\epsilon_{2}(\Gamma K)$ & $\gtrsim 10^{-3}$ & $1.5 \pm 0.1$ \\
\hline \hline
\end{tabular}



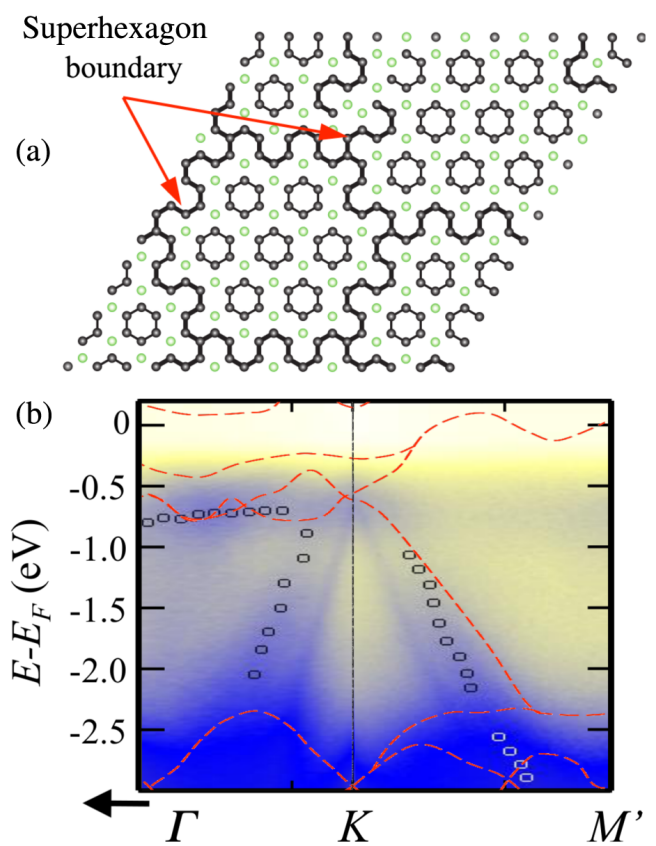

FIG. 5 (color online). (a) The calculated BG layer $(6 \sqrt{3} \times$ $6 \sqrt{3}) R 30^{\circ}$ cell on a relaxed $\mathrm{SiC}(0001)$ surface [20]. Green circles are buffer carbon atoms bonded to the SiC surface. Black circles are unbounded carbon. Chains of unbounded atoms define superhexagonal regions. (b) The calculated bands (dashed lines) from the structure in (a) overlaid on the ARPES bands. The theoretical bands are shifted $-0.13 \mathrm{eV}$ to match the $\epsilon_{2}$ band.

the $\mathrm{SiC}$ interface. The resulting structure is a hexagonal network of graphene ribbonlike structures with the remaining buffer carbon atoms covalently bonded to the $\mathrm{SiC}$ surface [see Fig. 5(a)]. Similar hexagonal networks, either structural or electronic, have been observed in scanning tunneling microscopy or produced theoretically [21-23]. The density-functional theory calculations show that the $\pi$ orbitals of carbon atoms on the superhexagonal boundaries give rise to several bands near the $K$ point above and below $E_{F}$. These bands are overlaid on our measured bands in Fig. 5(b). Like the experimental $\bar{\epsilon}_{1}(k)$ and $\epsilon_{2}(k)$ bands, the theoretical model shows that the covalent bonding to the $\mathrm{SiC}$ does not completely destroy the $\pi$ bands as earlier calculations predicted. Nonetheless, the calculations do not reproduce several important experimental features. The calculations do not predict the formation of a band gap, nor do they correctly reproduce the $\bar{\epsilon}_{1}$ dispersion, especially from $\Gamma$ to $K$.

We suggest that, while the model of Kim et al. [20] does not produce a band gap, there are features in the calculated structure that may lead to a band gap once a correct substrate interaction is taken into account. The large amount of covalent bonds associated with a bulk terminated $\mathrm{SiC}$ used in the calculation likely overestimates the graphene-SiC interaction. It is more likely that the buffer graphene is bonded to the $\mathrm{SiC}$ through a smaller number of sites consistent with STM measurements that suggest the buffer lies above a small set of Si trimers [24]. A reduced buffer-SiC bonding geometry is also consistent with both $\mathrm{x}$-ray scattering [25] and $\mathrm{x}$-ray standing wave experiments [26], which find a reduced Si concentration and an increased $\mathrm{C}$ concentration in the $\mathrm{SiC}$ layer below the buffer. We suggest that the reduced substrate bonding would still be sufficient to strain the buffer and produce the ribbon network. In fact, the ribbon network is the only structural part of the calculation that has been confirmed experimentally by STM [22]. However, the experimental width of the ribbons is about twice as wide as Kim et al. [20] predict. We postulate that the part of the buffer that is bonded to the $\mathrm{SiC}$ is so altered by the interaction that it becomes electronically decoupled from the ribbons. The ribbon network would now be isolated from the substrate and could give rise to a connected hexagonal graphene ribbon network instead of the linear chain of carbon atoms predicted in the model. The narrow ribbons would have a band gap due to the finite size effect [27]. Given the narrow ribbon width, finite size gaps are consistent with the size of the band gap we measure.

In this work, we show that, despite claims to the contrary, a semiconducting form of graphene can be manufactured. Using improved growth methods, we have been able to produce a well-ordered single layer of graphene on the $\mathrm{SiC}(0001)$ surface. The first graphene layer, known as the buffer layer, is a semiconducting form of graphene. Using ARPES, we show that the improved sample order leads to new bands with a band maximum that lies $\Delta E \sim 0.5 \mathrm{eV}$ below the Fermi level. Depending on where the conduction band lies, the band gap of this form of graphene must be $>0.5 \mathrm{eV}$. While no theoretical model predicts the measured bands, the experimental bands resemble those from a network of graphene ribbons that are distortions in the buffer layer. The distortions would be due to strain relief caused by a subset of carbon atoms in the buffer that locally bond to the SiC surface.

This research was supported by the NSF under Grants No. DMR-1401193 and No. DMR-1005880. M. S. N. also acknowledges support from the NSF PREM program under Grant No. DMR-0934142. Part of the work was funded by the French Research Agency under contract ANR Corigraph. Additional support came from the Partner University Fund from the French Embassy and the Keck foundation. The authors thank W. de Heer and A. Zangwill for many helpful discussions.

*edward.conrad@physics.gatech.edu

[1] T. Ohta, A. Bostwick, J. L. McChesney, T. Seyller, K. Horn, and E. Rotenberg, Phys. Rev. Lett. 98, 206802 (2007).

[2] K. V. Emtsev, F. Speck, T. Seyller, L. Ley, and J. D. Riley, Phys. Rev. B 77, 155303 (2008).

[3] C. Riedl, C. Coletti, T. Iwasaki, A. A. Zakharov, and U. Starke, Phys. Rev. Lett. 103, 246804 (2009). 
[4] F. Varchon et al., Phys. Rev. Lett. 99, 126805 (2007).

[5] A. Mattausch and O. Pankratov, Phys. Rev. Lett. 99, 076802 (2007).

[6] K. S. Novoselov, V. I. Fal'ko, L. Colombo, P. R. Gellert, M. G. Schwab, and K. Kim, Nature (London) 490, 192 (2012).

[7] E. Bekyarova, S. Sarkar, S. Niyogi, M. E. Itkis, and R. C. Haddon, J. Phys. D 45, 154009 (2012).

[8] S. Niyogi et al., Nano Lett. 10, 4061 (2010).

[9] M. Conrad et al. (to be published).

[10] C. Berger et al., J. Phys. Chem. B 108, 19912 (2004).

[11] C. Berger et al., Science 312, 1191 (2006).

[12] W. A. de Heer, C. Berger, M. Ruan, M. Sprinkle, X. Li, Y. Hu, B. Zhang, J. Hankinson, and E. Conrad, Proc. Natl. Acad. Sci. U.S.A. 108, 16900 (2011).

[13] K. V. Emtsev et al., Nat. Mater. 8, 203 (2009).

[14] A. Bostwick, T. Ohta, T. Seyller, K. Horn, and E. Rotenberg, Nat. Phys. 3, 36 (2007).

[15] E. McCann and V. I. Fal'ko, Phys. Rev. Lett. 96, 086805 (2006).

[16] T. Ohta et al., Science 313, 951 (2006).

[17] K. Okahara, K. Tanaka, H. Aoki, T. Sato, and T. Yamabe, Chem. Phys. Lett. 219, 462 (1994).
[18] S.-H. Lee, H.-J. Chung, J. Heo, H. Yang, J. Shin, U.-I. Chung, and S. Seo, ACS Nano 5, 2964 (2011).

[19] T. Schumann, M. Dubslaff, M. H. Oliveira, M. Hanke, J. M. J. Lopes, and H. Riechert, Phys. Rev. B 90, 041403 (R) (2014).

[20] S. Kim, J. Ihm, H. J. Choi, and Y. W. Son, Phys. Rev. Lett. 100, 176802 (2008).

[21] F. Owman and P. Mårtensson, Surf. Sci. 369, 126 (1996).

[22] C. Riedl, U. Starke, J. Bernhardt, M. Franke, and K. Heinz, Phys. Rev. B 76, 245406 (2007).

[23] F. Varchon, P. Mallet, J. Y. Veuillen, and L. Magaud, Phys. Rev. B 77, 235412 (2008).

[24] G. M. Rutter, N. P. Guisinger, J. N. Crain, E. A. A. Jarvis, M. D. Stiles, T. Li, P. N. First, and J. A. Stroscio, Phys. Rev. B 76, 235416 (2007).

[25] J. Hass, J. E. Millan-Otoya, P. N. First, and E. H. Conrad, Phys. Rev. B 78, 205424 (2008).

[26] J. D. Emery, B. Detlefs, H. J. Karmel, L. O. Nyakiti, D. Kurt Gaskill, M. C. Hersam, J. Zegenhagen, and M. J. Bedzyk, Phys. Rev. Lett. 111, 215501 (2013).

[27] M. Fujita, K. Wakabayashi, K. Nakada, and K. Kusakabe, J. Phys. Soc. Jpn. 65, 1920 (1996). 